\title{
Nuevas tendencias en la docencia del posgrado-especialización en medicina
}

\author{
New trends in postgraduate teaching-medicine specialization
}

\author{
Ana Cecilia Olascoaga-Mesía
}

\begin{abstract}
Resumen
El residentado médico es un período de intenso aprendizaje en servicio. Las nuevas tendencias en la educación médica involucran también al residentado médico y actualmente estamos presenciando su evolución. Estas nuevas tendencias suponen cambios en la organización de los programas y en el diseño de los programas de especialización basados en competencias, la innovación en estrategias didácticas y en estrategias de evaluación, el cuidado del clima de aprendizaje y la acreditación de programas. Se presenta un resumen de estas tendencias y se describen algunas experiencias nacionales e internacionales.
\end{abstract}

Palabras clave: Medicina, docencia, aprendizaje, residentado médico, tendencia.

\section{Abstract}

Medical residency is a period of intense learning in service. New trends in medical education also involve medical residents and we are currently witnessing its evolution. These new trends involve changes in the organization of programmes and in the design of competency-based specialization programs, innovation in teaching strategies and evaluation strategies, the care of the learning climate and the accreditation of programs. A summary of these trends is presented and some national and international experiences are described.

Keywords: Medicine, teaching, learning, medical residency, trend.

El residentado médico, desde su creación, fue enfocado como un período de intenso aprendizaje en servicio durante el cual el médico tenía la oportunidad de trabajar en un campo clínico guiado por un grupo de maestros, profundizar en el estudio y ganar experiencia en el manejo de pacientes con problemas de un área o especialidad médica ${ }^{(1)}$. Con el tiempo, los programas de residentado pasaron a estar compuestos por un número determinado de rotaciones alrededor de los servicios médicos de un hospital, en un lapso de tiempo que, al completarse, llevaba a la obtención de un título de especialista.

Debido al crecimiento poblacional, el incremento del número de facultades de medicina, del número de programas de residentado y de médicos residentes alrededor del mundo, en las últimas décadas surgieron las siguientes interrogantes:

- ¿Cómo garantizamos que el médico que egresa de un programa de residentado esté preparado como un especialista idóneo en su campo, que pueda desenvolverse adecuadamente?

- ¿Cómo asegurar que todos los residentes de un programa reciban un entrenamiento similar con las mismas oportunidades de aprendizaje?
- ¿Estamos seguros que todos los residentes tuvieron suficientes y efectivas experiencias prácticas para certificar su aprendizaje?

- ¿Estamos seguros que estamos formando especialistas íntegros que no solo saben de su especialidad sino que actúan con ética y profesionalismo?

- ¿Estamos seguros de que en ese entrenamiento no pusimos en riesgo la salud de los pacientes?

- ¿Estamos seguros de que durante ese entrenamiento el residente no vio afectada su vida y su bienestar personal por la sobrecarga de trabajo y en un medio inadecuado y no pocas veces hostil?

Para dar respuesta a estas interrogantes, se observa que las nuevas tendencias en educación médica involucran al residentado médico en sus propuestas y, aunque no es fácil introducir cambios rápidamente, estamos presenciando la evolución de los programas de residentado en sus distintos niveles. 
Como todo programa educativo, la residencia médica necesita un diseño apropiado que, como afirma Jason R. Frank (2): "empieza pensando en el final"; es decir, que el diseño debe partir de la concepción de un perfil del egresado, un perfil que abarque tanto el ser experto en su especialidad médica como el ser un buen profesional, preparado para afrontar las exigencias de la sociedad en el mundo real. El programa de residentado debe definir las competencias que el estudiante tiene que adquirir año tras año de manera progresiva hasta culminar su entrenamiento, debe planificar las actividades y estrategias educativas que se llevarán a cabo durante el entrenamiento y, finalmente, debe indicar las formas de evaluación que garanticen que se lograron estas competencias ${ }^{(3)}$.

De estos puntos se desprenden las nuevas tendencias en el residentado médico, algunas de las cuales no son tan nuevas pero que en la realidad nacional, en general, podemos decir que los cambios propuestos por las tendencias todavía no han sido incorporados en todos los programas de residentado médico.

A partir de la revisión, se plantea una propuesta innovadora para la elaboración de programas de residentado médico considerando los siguientes puntos:

\section{Programas de residentado médico}

a. Organización de un programa de residentado médico.

b.Diseño de un programa por competencias / profesionalismo e identidad profesional /educación interprofesional / comunicación / residentes como docentes / telemedicina.

c. Estrategias didácticas/simulación/e-learning

d.Evaluación / feedback.

2. Clima de aprendizaje y bienestar del residente / residente en dificultad/remediación

3. Acreditación de los programas de residentado médico oresidencias

\section{Programas de Residentado Médico}

\section{a. Organización de un programa de residentado médico}

El primer paso para hacer un programa fuerte y eficiente es contar con un jefe de programa que diseñe, organice y evalúe el programa periódicamente. El jefe de programa, quien es un especialista experto en su campo, debe ser un profesional con capacidad de liderazgo y capacidad de solucionar conflictos y debe ser consciente de la importancia del papel que desempeña

(4). Un programa irá bien y crecerá en el tiempo si cuenta con un jefe de programa que se lo proponga y trabaje fuertemente con los profesores y los residentes. El trabajo del jefe de programa es reconocido como fundamental y complejo, muestra de ello es la creación en Estados Unidos del National Institute for Program Director Development desde 1994 y la existencia de múltiples recursos bibliográficos destinados a apoyarlo en su labor ${ }^{(5)}$. El Royal College of Physicians and Surgeons of Canada menciona, como uno de los requisitos de acreditación de programas, que el jefe de programa debe contar con el apoyo de un equipo denominado Comité de Especialidad, constituido por un grupo de profesores de las distintas sedes docentes y representantes de los residentes, quienes trabajan en conjunto en la planificación, organización y supervisión del programa mediante reuniones periódicas ${ }^{(6)}$.

\section{b.Diseño de un programa por competencias}

El diseño de un programa basado en competencias brinda importancia a la formación integral, facilita el desarrollo de las actividades significativas para lograr las metas esperadas y conduce hacia una evaluación eficiente del residente ${ }^{(7)}$. Existen diversos modelos de competencias a nivel mundial, los más reconocidos son: Canmeds ${ }^{(8)}, \mathrm{ACGME}^{(9)}$ y Scotish Doctor ${ }^{(10)}$. Estos modelos coinciden en perfilar al médico no solo como un experto en medicina sino también como un buen profesional, un comunicador, un gestor, un docente, un estudioso, un miembro de un equipo y un líder. Un grupo de educadores médicos, The International CBME Collaborators, resumió los cuatro principios del diseño de programas por competencias $^{(11)}: 1$. Enfocarse en los resultados, 2. Enfatizar las habilidades del alumno (no solo el conocimiento), 3. Prestar menor atención al tiempo como resultado primario del entrenamiento y 4. Promover el aprendizaje centrado en el estudiante.

Para empezar a diseñar un programa de residentado médico por competencias lo más recomendable es tomar uno de los modelos antes citados y aplicarlo a nuestra realidad en base a un análisis de la situación local, conociendo las necesidades de nuestra sociedad y de nuestros médicos. Este trabajo recae en el jefe de programa y el Comité de Especialidad, quienes posteriormente al diseño deben socializar el perfil de competencias entre los profesores buscando un consenso. Seguidamente, se deben definir las estrategias didácticas para lograr las competencias del perfil y las estrategias de evaluación.

El enfoque por competencias en la educación del residente ha permitido, por ejemplo, haber empezado a incluir el entrenamiento en habilidades de comunicación, que implica también la comunicación de malas noticias y la comunicación del error médico. Existen muchas experiencias en el mundo, en especialidades clínicas y quirúrgicas ${ }^{(12-14)}$ y en el Perú, en la Universidad Peruana Cayetano Heredia se ha empezado a entrenar a los residentes en habilidades de comunicación y en dar malas noticias con el uso de simulación y de guías existentes para tal fin, como las guías CICAA $^{(15)}$ y SPIKES $^{(16)}$, obteniendo altos índices de satisfacción entre los residentes. Los resultados fueron presentados en el reciente Congreso de Educación Médica de ASPEFAM Lima-2019.

El enfoque por competencias ha permitido también generar espacios para la educación interprofesional, espacios 
en los que confluyen alumnos de diversas áreas de la salud quienes comparten objetivos educacionales, trabajan juntos y aprenden unos de otros. En estos espacios se refuerza el trabajo en equipo, en un clima de respeto y colaboración ${ }^{(17)}$. Se ha demostrado que la educación interprofesional mejora la calidad del servicio prestado a los pacientes y disminuye el error médico ${ }^{(18-19)}$. Las experiencias publicadas se presentan en espacios simulados y en espacios reales. En nuestro país no hemos encontrado publicaciones al respecto; sin embargo, en nuestra facultad se vienen realizando sesiones pilotos de entrenamiento en el manejo de situaciones críticas de emergencia en ambientes de simulación con grupos de residentes de medicina y de enfermería.

Proveer a los residentes de herramientas para desarrollar competencias en telemedicina es otro ejemplo que responde a las competencias necesarias para un especialista en la actualidad; así, existen ejemplos publicados en psiquiatría, neurología, dermatología y otras especialidades más ${ }^{(20-22)}$. Gran parte de las regiones en el país carece de especialistas, siendo una necesidad nacional el contar con especialistas preparados en telemedicina para poder solucionar problemas médicos que se presentan en lugares remotos con pobre acceso a la atención médica y para telecapacitar a otros profesionales de la salud. Las universidades nacionales vienen realizando esfuerzos por introducir este aspecto en los currículos del residentado médico con cursos virtuales y semipresenciales y de introducir a los residentes en los sistemas de telesalud existentes en las distintas sedes docentes.

El profesionalismo es definido por el Royal College of Physicians of UK como un conjunto de valores, comportamientos y relaciones que sustentan la confianza que el público tiene en los médicos. En el 2002 el American Board of Internal Medicine publicó el Charter of Medical Professionalism que incluye nueve responsabilidades: compromiso con la competencia profesional, honestidad con los pacientes, confidencialidad, compromiso con mantener relaciones apropiadas con los pacientes, compromiso con brindar un cuidado de calidad, compromiso con mejorar el acceso a la salud, compromiso con el conocimiento científico, compromiso con las responsabilidades profesionales y compromiso con el manejo de los conflictos de interés ${ }^{(23)}$. Si bien es totalmente aceptado que el profesionalismo es intrínseco a toda práctica médica, es difícil integrarlo al currículo formal $^{(24)}$.

El primer paso para desarrollar un marco curricular de profesionalismo es garantizar que todos los profesores sean partícipes de estos conceptos ${ }^{(25)}$. El profesionalismo puede y debe ser entrenado, aprendido y evaluado. Existen diversas estrategias educativas para la enseñanza-aprendizaje del profesionalismo, pero en todas ellas el $\mathrm{AMEE}^{(25)}$ recomienda la aplicación del Ciclo de Aprendizaje de Kolb que consiste en:

- Planeamiento: definir el caso en base a un marco conceptual.

- Experiencia concreta: visualización de videos o películas, enfrentar un caso difícil en simulación, presentar un caso real.

- Conceptualización abstracta: discusión grupal, feedback, conceptos, sentimientos, actitudes.

- Observación reflexiva: el tutor presenta guías para la práctica, une la práctica con la teoría.

- Experimentación activa: el alumno concluye con ¿qué aprendí? y ¿cómo haría la próxima vez que tenga un caso similar?

La evaluación del profesionalismo se debe realizar tomando en consideración la información vertida no solo por los profesores sino también por los pares, los residentes superiores, por los internos y alumnos, por las enfermeras y técnicas, e incluso por los pacientes, en lo que se denomina la evaluación $360^{\circ}$. Y, finalmente, los residentes que tienen deficiencias en esta competencia deben tener la guía o tutoría de un mentor y la oportunidad de una remediación. Existen algunas experiencias publicadas en Latinoamérica pero aún falta mucho por hacer en el residentado médico en este campo (26-28)

La competencia docente en el médico residente es reconocida desde hace décadas. El médico residente cumple un rol docente durante su entrenamiento enseñando a residentes menores, internos y alumnos. Existen múltiples publicaciones de experiencias de entrenamiento a residentes, de distinta duración y variable contenido. Algunas experiencias de la Universidad Católica de Chile y de nuestra facultad de medicina presentan intervenciones que demuestran una mejoría en la autopercepción de las capacidades docentes de los propios residentes y la aplicación de lo aprendido en sesiones en grupo pequeño, mejorando el clima de aprendizaje, brindando retroalimentación (feedback) y promoviendo el autoaprendizaje $\mathrm{e}^{(29,30)}$. Actualmente, constituye un reto el incluir este tipo de capacitación a todos los residentes en formación pero se deben buscar las estrategias que lo permitan.

\section{c. Estrategias didácticas}

El aprendizaje de procedimientos durante la residencia tradicionalmente se realiza durante la práctica en servicio, inicialmente observando, luego ayudando, ejecutando con apoyo y finalmente ejecutando independientemente. Esta forma de aprendizaje se ve afectada por la oportunidad y la capacidad del tutor y, por otro lado, pone en riesgo la seguridad del paciente.

En los últimos años, cada vez más se viene haciendo uso de la simulación como herramienta docente que permite enseñar a realizar procedimientos en un ambiente seguro. La simulación en el residentado médico ha tomado un rol importante tanto en especialidades clínicas como quirúrgicas, permite entrenar en procedimientos diversos y de variada complejidad en casi todas las especialidades. El uso de la simulación ha demostrado mejorar las habilidades de los residentes dentro de sala de operaciones, en habilidades procedimentales, en habilidades clínicas, así como en comunicación y trabajo en equipo ${ }^{(31)} \mathrm{y}$, sobre todo, la experiencia en simulación es altamente valorada por los 
residentes porque incrementa la seguridad en sí mismos ${ }^{(32)}$. Hay muchas experiencias internacionales publicadas. En Perú, a pesar de que sabemos que se han creado muchos centros de simulación y se ha empezado a utilizar esta herramienta en distintas facultades, aún no encontramos muchos trabajos publicados; así, resalta el trabajo sobre entrenamiento a residentes de ginecología en manejo de distocia de hombros ${ }^{(33)}$ y la presentación de la evaluación del curso de procedimientos para residentes de clínicas médicas en el Congreso LACRE (Latin American Conference on Residency Education) 2019 ${ }^{(34)}$, ambos realizados en la Universidad Peruana Cayetano Heredia.

Es seguro que el uso de la simulación se va a ir incrementando conforme pase el tiempo; por tanto, es pertinente mencionar que el uso de la simulación tiene que responder a objetivos educacionales claramente trazados; contar con profesores entrenados, con un diseño de curso y con listas de cotejo adecuadamente diseñadas; realizar debriefing (evaluación post actividad) correctamente; hacer una evaluación de cada residente y garantizar el seguimiento de su desempeño una vez terminada la intervención. Los jefes de programa deben estar atentos a los resultados a largo plazo de las intervenciones en simulación, deben preocuparse por medir el impacto de estos cursos, su utilidad en el aprendizaje y el beneficio para los pacientes.

E-learning, o electronic learning, se refiere al uso de tecnologías de internet como herramienta de aprendizaje. $\mathrm{Su}$ uso durante el residentado se ha extendido alrededor del mundo debido a que aporta la facilidad de llegar a todos los residentes, sobre todo cuando se tienen programas de residentado que se desarrollan en varias sedes docentes y los residentes están distribuidos por toda la ciudad. Así mismo, brinda flexibilidad de horarios, se adapta al ritmo de aprendizaje de cada estudiante, asegura el control sobre el acceso a los espacios virtuales y permite realizar evaluaciones. Los docentes pueden colocar en los espacios virtuales el material informativo que puede ser revisado virtualmente, se pueden utilizar lecturas, clases grabadas y videos, y también se pueden generar foros de discusión. Aporta la ventaja de que el residente puede revisar el material y participar en los foros (que son el equivalente a discutir un tema en clase presencial), dar los exámenes de acuerdo con el horario disponible y en el lugar en que se encuentre, sin tener que desplazarse en la ciudad y salir de su hospital.

El uso de los espacios virtuales en el residentado médico está ampliamente difundido en Estados Unidos ${ }^{(35)}$, más no contamos con una revisión de la extensión de su uso a nivel nacional. El uso de espacios virtuales no obstante demanda tiempo y esfuerzo al cuerpo docente que tiene que encargarse del diseño de los cursos, de la producción del material didáctico, de revisar y dirigir los foros, de vigilar la participación de los alumnos y monitorizar las evaluaciones. En nuestra universidad, el área de especialización de nuestra facultad tiene una experiencia con los cursos de salud mental, metodología de la investigación, comunicación y procedimientos, que tienen una parte de e-learning.

\section{d. La evaluación}

La evaluación en el residentado médico también ha dado un cambio sustancial. Luego de largos años acostumbrados a una calificación mensual en conocimientos, habilidades y actitudes en base a una escala del 0 al 20, totalmente subjetiva y cargada de múltiples sesgos, surgen otros métodos de evaluación apropiados para cada área a evaluar y que corresponden a cada nivel de la Pirámide de Miller y adecuados al nivel de formación de cada alumno, este es, no debemos esperar el mismo nivel de competencias en un residente de primer y otro de tercer año.

Describiré brevemente los métodos de evaluación más representativos por área a evaluar. Para el área de conocimientos siempre estará a la cabeza el examen de conocimientos, examen escrito con preguntas de opción múltiple o con preguntas abiertas, realizadas con una redacción y un diseño apropiado basado en una tabla de planificación. En el residentado médico se deben enfocar las preguntas hacia el análisis y síntesis y a la resolución de problemas; además, la mayoría de las preguntas deben ser casos clínicos. The National Board of Medical Examiners ha creado una guía excelente para elaborar preguntas ${ }^{(36)}$.

Para evaluar habilidades y destrezas se despliega un amplio abanico de posibilidades: la ficha de evaluación en base a la escala de Likert al final de la rotación (Global Rating Scale); el uso de rúbricas para habilidades complejas como la presentación de un caso clínico, la redacción de notas de ingreso y la lectura crítica de un artículo científico; la lista de cotejo para la realización de procedimientos; el registro de procedimientos en repositorios o procedural (logbooks) para llevar la cuenta de los procedimientos realizados por los residentes, el número y la capacidad de realización (si los realizaron solos o con ayuda). El Mini Clinical Examination merece mención aparte, pues es un método útil para evaluar el cuarto nivel de la Pirámide de Miller: el desempeño del residente en el ambiente de trabajo -un área de emergencia, hospitalización o consulta externa- donde se evalúa la anamnesis, el examen físico, el juicio clínico, organización y eficiencia, profesionalismo, habilidades de comunicación y la valoración global; fue creado por el American Board of Physicians en Estados Unidos y actualmente está difundido en todo el mundo debido a su practicidad y a haber demostrado ser un instrumento altamente confiable ${ }^{(37)}$. En nuestro país, su uso ha sido introducido con éxito en el programa de medicina interna de la UPCH desde el año 2014, permitiendo la retroalimentación y la mejora progresiva de las competencias clínicas de los residentes ${ }^{(38)}$.

Para el área actitudinal puede aplicarse el Global Rating Scale, pues consigna todas las competencias actitudinales esperadas y las mide en una escala de Likert; sin embargo, para evaluar esta área, la evaluación de $360^{\circ}$ es una de las más apropiadas. Esta es una evaluación estructurada que se centra en las competencias: comunicación, trabajo en equipo y profesionalismo; evalúa el cuarto nivel de la Pirámide de Miller que es el desempeño en el lugar de trabajo. Es realizada por los 
profesores médicos, los residentes superiores, los pares, los internos, las enfermeras, las técnicas, los pacientes quienes se encuentran alrededor del residente, e incluye la autoevaluación del residente; de esta manera, se disminuye la subjetividad. Este método proviene del área empresarial. Se recomienda que el instrumento que se diseñe se confeccione con detenimiento y se valide. Una vez obtenidos los resultados, la retroalimentación es fundamental. Este método es esencialmente para la evaluación formativa pero puede ser usado también para la evaluación sumativa ${ }^{(3)}$.

Diego Correa, médico de la Pontificia Universidad Católica de Chile, diseñó y validó un instrumento $360^{\circ}$ basado en competencias CanMEDS ${ }^{(39)}$. Se trata de una ficha de 15 ítems con una escala de Likert del 1 al 9, de muy fácil aplicación y que está siendo utilizada actualmente en los programas de residentado médico de nuestra facultad. El portafolio es otro método apropiado para la evaluación principalmente formativa de las competencias actitudinales de los residentes, y se ubica en el cuarto nivel de la Pirámide de Miller. Se define como la "recolección más o menos sistemática de elementos que evidencian el aprendizaje o nivel de competencia conseguido durante un período de tiempo, según unos objetivos previamente establecidos y todo ello acompañado de un proceso de reflexión"(3). La reflexión es lo que marca la diferencia entre un portafolio y un logbook o registro de actividades. Para su diseño el primer paso es definir el propósito y elegir las competencias que se van a desarrollar (este método es especialmente útil para la enseñanzaaprendizaje del profesionalismo). Su uso implica una inversión significativa de tiempo del residente y del tutor $\mathrm{y}$, por tanto, se debe valorar su uso con relación al número de residentes de un $\operatorname{programa}^{(40)}$.

Finalmente, es necesario recalcar que la evaluación en el residentado debe ser continua, multidimensional, multimétodo y basada en competencias. Además, el efecto formativo de la evaluación se produce cuando esta va seguida de una retroalimentación ofeedback hecha por el profesor.

La World Federation of Medical Education, en su documento Estándares Globales en la Educación Médica ${ }^{(41)}$, considera que, en el posgrado, la retroalimentación o feedback debe realizarse regularmente en base a los resultados de las evaluaciones y la observación directa. Se debe capacitar a los docentes en brindar feedback a los alumnos y se debe destinar un tiempo protegido para tal fin. Hemos tenido la experiencia de introducir un método de retroalimentación en un grupo de profesores en una sede hospitalaria nacional ${ }^{(42)}$.

\section{Clima de Aprendizaje}

En los últimos años ha cobrado importancia la evaluación del clima de aprendizaje. Clima de aprendizaje o ambiente educacional se refiere a todo lo que ocurre alrededor del proceso de enseñanza-aprendizaje: el programa curricular, los profesores, los compañeros, las sedes hospitalarias. El ambiente educacional es un gran determinante de la conducta de los alumnos y, por lo tanto, de su aprendizaje ${ }^{(3)}$. El ambiente educacional ha sido relacionado positivamente con el bienestar del residente ${ }^{(43)}$, con el desempeño de los tutores de residentado ${ }^{(44)}$, con la experiencia de los pacientes hospitalizados ${ }^{(45)} \mathrm{y}$, negativamente, con el burnout ${ }^{(46)}$.

Se han creado diversos instrumentos para medir el ambiente educacional y existen instrumentos específicos para el residentado médico. El instrumento PHEEM $^{(47)}$ está siendo validado en nuestra facultad para regularizar su uso y promover las buenas prácticas docentes estableciendo las medidas correctivas correspondientes.

El residentado es un momento en la vida del médico que representa un reto y puede llegar a ser muy difícil debido a las exigencias educativas y asistenciales que confluyen en este período, además de la existencia reconocida de maltrato en gran parte de los programas de residencia alrededor del mundo ${ }^{(48)}$. Es un momento en el que los residentes suelen presentar reacciones de estrés, burnout y trastornos del ánimo, pudiendo estos llevar hacia el fracaso y el abandono del programa académico por parte del residente. Es por ello que es necesario prestar atención a esta situación de vulnerabilidad y brindar el apoyo necesario. El concepto de bienestar del residente toma un lugar esencial en la actualidad y, en ese sentido, se vienen generando, en las facultades, las unidades de apoyo al residente y coordinaciones de bienestar que trabajan en la instauración de medidas preventivas como el entrenamiento en resiliencia, la detección temprana de residentes en dificultad y el seguimiento y la resolución de los casos individuales ${ }^{(49)}$. El Área de Especialización de nuestra facultad ha creado, este año, la Coordinación de Profesionalismo y Bienestar para llevar a cabo estas acciones.

\section{Acreditación de los Programas de Residentado Médico}

La acreditación es un proceso voluntario al cual se somete un programa educativo para ser evaluado por una entidad externa que en base a ciertos criterios establecidos determina sus niveles de calidad. La acreditación tiene como unos de los objetivos esenciales el orientar los programas hacia la mejora continua y el logro de la excelencia.

Dada la complejidad del sistema de residentado médico, que es un sistema educativo que se desarrolla completamente en sedes hospitalarias, resulta complejo llevar a cabo el proceso de acreditación. Actualmente, CONAREME se prepara para desarrollar el proceso de acreditación de sedes docentes con la finalidad de reconocer a los centros con un nivel de calidad adecuado para la formación de especialistas y subespecialistas en medicina.

Sin embargo, la acreditación de programas de residentado es un proceso al que debe someterse cada uno de los programas académicos de residentado de las distintas facultades de medicina y esto representa una inversión importante de esfuerzo, tiempo y dinero. 
El marco conceptual de un proceso de acreditación de programas de residentado médico se basa en tres puntos ${ }^{(50)}$ :

1. Estructura: Objetivos, planificación, organización, recursos humanos e infraestructura.

2. Proceso: Desarrollo y cumplimiento del currículo, actividades y evaluación.

\section{Resultado: Médicos especialistas competentes.}

Existen diversas experiencias internacionales en acreditación de programas de residentado, la más reconocida es el sistema de Acreditación del Royal College of Physicians and Surgeons of Canada, que además de evaluar los programas de residentado de Canadá ha definido los criterios para la acreditación de programas internacionales y viene evaluando distintos programas de residentado médico en Latinoamérica.

\section{Conclusión}

Existen muchas innovaciones en la docencia en posgrado, con evidencia demostrada de efectividad, pero su aplicación en los programas nacionales viene sucediendo lenta y progresivamente. Es necesario, para ello, la unión de esfuerzos académicos y económicos. Esperamos que con el tiempo podamos ver la aplicación y los resultados de estas innovaciones en el residentado médico a nivel local y nacional.
Referencias bibliográficas

1. Howell JD. A History of Medical Residency. Reviews in American History. 2016;44(1):126-131.

2. Sherbino J, Frank Jr. A CanMeds guide for the health professions. Chapter 1. Ottawa: Royal College of Physicians and Surgeons; 2011.

3. Millán J, Palés J, Morán-Barrios J. Principios de educación médica, desde el grado hasta el desarrollo profesional. Capítulo 9. Planificación curricular. Etapas de la planificación curricular. Editorial Médica Panamericana; 2015.

4. Harris K, Chapter 2. Creating the future for your program. En: The Royal College program directors handbook: A practical guide for leading an exceptional program; 2014.

5. Pugno P. The National Institute for Program Director Development: a school for program directors. The Journal of the American Board of Family Practice. 2002;15(3)209-213.

6. Royal College of Physician and Surgeons of Canada. International Program Standars. 2014.

7. Harden RM, Laidlaw JM. Essential skills for a medical teacher: An introduction to teaching and learning in medicine. Section 2. Elsevier; 2017.

8. Frank JR. The CanMEDS 2005 physician competency framework. Better standards. Better physicians. Better care. Ottawa: The Royal College of Physicians and Surgeons of Canada, 2005.

9. Accreditation Council for Graduate Medical Education. Implementing milestones and clinical competency committees. URL disponible en:http:/www.acgme.org/acgmeweb/Portals/ 0/PDFs/ACGMEMilestones-CCC-AssesmentWebinar.pdf.

10. Simpson JG, et al. The Scottish doctor learning outcomes for the medical undergraduate in Scotland: a foundation for competent and reflective practitioners. Medical Teacher. 2002; 24(2):136-143.

11. Frank JR, Snell LS, ten Cate O, Holmboe ES, Carraccio C, Swiing SR, et al. Competency-based medical education: theory to practice. Med Teach. 2010;32(8):638-645.

12. Karam et al. Effect of a simulation-based workshop on breaking bad news for anesthesiology residents: an intervention study. BMC Anesthesiology. 2017;17(1):77-83.

13. Hilkert SM, Cebulla CM, Jain SG, Pfeil SA, Benes SC, Robbins SL. Breaking bad news: a communication competency for ophthalmology training programs. Surv
Ophthalmol. 2016; 61(6):791-798.

14. Vellutini MS, Vasconcelos A, Ribeiro S, Martorano E. Breaking bad news training program based on video reviews and SPIKES Strategy: What do perinatology residents think about it? Rev Bras Ginecol Obstet. 2017;39:552-559.

15. Gavilán E, Ruiz R, Perula de Torres LQ, Parras JM. Valoración de la relación clínica centrada en el paciente: análisis de las propiedades psicométricas de la escala CICAA. Atención Primaria. 2010;42(3):162-168.

16. Baile WFE, Buckman R, Lenzi R, Glober G, Beale E, Kudelka AP. SPIKES - A six-step protocol for delivering bad news: application to the patient with cancer. The Oncologist, 2000;5:302-311.

17. Al Achkar M, Mathew Hanauer, Colavecchia CH, Seehusen DA. Interprofessional education in graduate medical education: survey study of residency program directors. BMC Medical Education. 2018;8:11-16.

18. Reeves S, Perrier L, Goldman J, Freeth D, Zwarenstein M. Interprofessional education: effects on professional practice and healthcare outcomes (update). Cochrane Database Syst Rev. 2013;(3):CD002213.

19. Reeves $S$, et al. A BEME systematic review of the effects of interprofessional education: BEME Guide No. 39. Med Teach. 2016;38(7):656-668.

20. Crawford A, Sunderji N, López J, Soklaridis S. Defining competencies for the practice of telepsychiatry through an assessment of resident learning needs. BMC Medical Education. 2016;16:28-37.

21.Jagolino AL, et al. A call for formal telemedicine training during stroke fellowship. Neurology. 2016;86(19):1827-1833.

22. Nelson CA, Wanat KA, Roth RR, James WD, Kovarik CL, Takeshita J. Teledermatology as pedagogy: diagnostic and management concordance between resident and attending dermatologists. J Am Acad Dermatol. 2015;72(3):555-557.

23. Project of the ABIM Foundation, ACP-ASIM Foundation and European Federation of Internal Medicine. Medical Professionalism in the New Millennium: A Physician Charter. Ann Intern Med. 2002;136:243-246.

24. Pinilla AE, Cárdenas FA. Evaluación y construcción de un perfil de competencias profesionales en medicina. Acta Med Colomb. 2014;39(2):165-154. 
25. O'Sullivan H, van Mook W, Fewtrell R, Wass V. Integrating professionalism into the curriculum. Medical Teacher. 2012;34(2):e64-e77.

26. Grenno A, et al. Formación de médicos en postgrado de ginecología mediante entrenamiento simulado. Horiz Med. 2014;14(3):6-11.

27. Gonzáles MI, Garmendia C, Moreno L. Una estrategia para la formación en seguridad del paciente durante la residencia: desde el incidente crítico hasta la simulación. Parte 1. Educ Med. 2019;20(3):170-178.

28. Cordero Díaz MA, Guerra D, Arce CF, Dávila Rivas JA. Programa de profesionalismo y bienestar para médicos residentes. Instituto Tecnológico y de Estudios Superiores de Monterrey. Escuela Nacional de Medicina del Tecnológico de Monterrey. Resúmenes LACRE 2017. Investigación en Educación Médica. 2017;6,(22):e1-e48,67-144.29.

29. Reyes C, Florenzano P, Contreras Á, González A, Beltrán D, Aravena C, et al. Un curso de docencia clínica para residentes mejora la autopercepción de preparación para enseñar. Rev Med Chile. 2012;140:1431-1436.

30. Olascoaga AC. Curso de docencia para residentes: evaluación de un programa. Educ Med. 2018. https://doi.org/10.1016/ j.edumed.2018.07.010

31. Okuda Y, et al. The Utility of Simulation in Medical Education: What Is the Evidence? Mount Sinai Journal of Medicine. 2009;76:330-343.

32. Yamamoto A, et al. Effects on postgraduate-year-I residents of simulation-based learning compared to traditional lecturestyle education led by postgraduate-year-II residents: a pilot study. BMC Medical Education. 2019;19:87-97.

33. Flores M, Alfaro L. Resultados de la aplicación de un curso taller de distocia de hombros utilizando simulación en el programa de residentado médico de Obstetricia y Ginecología de la Universidad Peruana Cayetano Heredia. Educ Med. 2019;20(3):162-66.

34. Olascoaga A, García A, Mas G. Evaluación de la implementación de un Curso de Procedimientos para Residentes. Libro de resúmenes, IV Latin American Conference on Medical Education LACRE 2019. ARS Médica. Revista de Ciencias Médicas. 2019;44 (Supp 1).

35. Wittich CM, et al. E-learning in graduate medical education: survey of residency program directors. BMC Medical Education. 2017;17:114-120.

36. National Board of Medical Examiners. Cómo elaborar preguntas para evaluaciones escritas en las áreas de ciencias básicas y clínicas. Philadelphia; 2016.

37. Fornells-Valles JM. El ABC del Mini-CEx. Educ Med.
2009;12(2):83-89.

38. Olascoaga AC, Riquelme A. Aplicación longitudinal del Mini Clinical Examination (Mini-CEX) en médicos residentes. Educación Médica. 2019;20(Suppl 1):25-28.

39. Correa D, Triviño X, Gonzáles C, Abarca K. Diseño de un instrumento de evaluación en $360^{\circ}$ para residentes de la Pontificia Universidad Católica de Chile, basado en competencias CanMeds. Investigación en Educación Médica. 2017;6(22):e1-e48,67-144.

40. Schlatter J. Experiencias del uso del portafolio para residentes de la Clínica Universidad de Navarra. FEM. 2013;16(1):59-62.

41. WFME. Estándares Globales en la Educación Médica Educación Médica. 2004;7(Suppl 2):1-56.

42. Olascoaga A, Aphang M. Retroalimentación en docencia médica. Rev Soc Peru Med Intern. 2017;30(3):172-175.

43. Lases SS, Arah OA, Busch ORC, Heineman MJ, Lombarts K. Learning climate positively influences residents' workrelated well-being. Adv Health Sci Educ Theory Pract. 2019;24 (2):317-330

44. Lombarts K, Heineman MJ, Scherpbier AJ, Arah OA. Effect of the learning climate of residency programs on faculty's teaching performance as evaluated by residents. PLoS One. 2014;28;9(1):e86512.

45. Smirnova A1, Arah OA, Stalmeijer RE, Lombarts KMJMH, van der Vleuten CPM. The association between residency learning climate and inpatient care experience in clinical teaching departments in the Netherlands. Acad Med. 2019;94(3):419-426.

46. van Vendeloo SN, Brand PL, Verheyen CC. Burnout and quality of life among orthopaedic trainees in a modern educational programme: importance of the learning climate. Bone Joint J. 2014;96-B(8):1133-1138.

47. Clapham M, Wall D, Batchelor A. Educational environment in intensive care medicine - use of Postgraduate Hospital Educational Environment Measure (PHEEM). Medical Teacher. 2007;29(6):e184-191.

48. Fnais $\mathbf{N}$, et al. Harassment and Discrimination in Medical Training: A Systematic Review and Meta-Analysis. Academic Medicine. 2014;89(5):817-827.

49. Cordero MA, González M, De la Rosa C. Programa de Bienestar de Residentes: Orientación y Evaluación Inicial. Libro de resúmenes IV Latino American Conference on Medical Education LACRE 2019. ARS Médica. Revista de Ciencias Médicas. 2019;44(Supp 1).

50. Antunes R, Snell L, do Patrocinio M. The link between quality and accreditation of residency programs: the surveyors' perceptions. Medical Education Online. 2017;22(1):1270093.

Citar como: Olascoaga-MesíaAC. Nuevas tendencias en la docencia del posgrado-especialización en medicina. Diagnóstico (Lima). 2019;58(3):125-131. DOI: $10.33734 /$ diagnostico.v58i3.58

Correspondencia: Ana Cecilia Olascoaga-Mesía Correo electrónico: ANA.OLASCOAGA.M@upch.pe 